\title{
DESENVOLVIMENTO DE TACÔMETRO MAGNÉTICO PARA MEDIÇÃO DE VELOCIDADE EM PIGs INSTRUMENTADOS
}

\author{
Antônio Eduardo de Morais Silva*, Gustavo Fernandes de Lima, Andrés Ortiz \\ Salazar \\ Universidade Federal do Rio Grande do Norte \\ Laboratório de Avaliação de Medição em Petróleo \\ *edumorais92@gmail.com
}

Submetido 10/10/2016 - Aceito 02/03/2017

DOI: $10.15628 /$ holos.2017.5202

\begin{abstract}
RESUMO
A inspeção de dutos e tubulações é preocupação constante das empresas transportadoras de petróleo e derivados, visando a prevenção de vazamentos e desastres ambientais. Para esta tarefa são utilizadas ferramentas de sensoriamento interno, chamadas de PIGs instrumentados, para a constatação da integridade dessas tubulações. Este trabalho apresenta os detalhes do desenvolvimento de um tacômetro magnético, utilizando um microcontrolador ATMEGA328P e um sensor de efeito Hall modelo 3144. Um protótipo foi
\end{abstract}

\begin{abstract}
construído com uma roda de $66 \mathrm{~mm}$ de diâmetro e em sua lateral foi fixado um ímã de neodímio. A cada passagem do ímã pelo sensor é calculada a velocidade pelo microcontrolador. As leituras de velocidade obtidas pelo foram comparadas com as leituras de um tacômetro óptico, com erro menor do que $0,5 \%$. Os resultados mostraram que é possível construir, de forma bem sucedida, um tacômetro magnético para a determinação da velocidade de um PIG instrumentado.
\end{abstract}

PALAVRAS-CHAVE: PIG, tacômetro, sensor de efeito Hall, microcontrolador, prototipagem.

\section{DEVELOPMENT OF MAGNETIC TACHOMETER FOR SPEED MEASUREMENT IN SMART PIG'S}

\begin{abstract}
Inspection in ducts and pipelines is a constant concern of oil and derivatives carriers companies which aim to prevent spills and environmental disasters. For this task, internal sensing tools called smart PIGs are used to verify the integrity of these pipelines. This work features details of the development of a magnetic tachometer using an ATMEGA328P microcontroller and a model 3144 Hall Effect sensor. A prototype was built with a $66 \mathrm{~mm}$ diameter wheel and a
\end{abstract}

neodymium magnet was fixed to the side. Each time the magnet passes through the sensor, the speed is calculated by the microcontroller. The velocity readings obtained were compared with readings of an optical tachometer, with error less than $0.5 \%$. The results showed that it is possible to construct, in a very successful way, a magnetic tachometer to determine the velocity of smart PIGs.

KEYWORDS: PIG, tachometer, Hall effect sensor, microcontroller, prototyping. 


\section{INTRODUÇÃO}

O Pipeline Inspection Gauge (PIG) é uma ferramenta utilizada em dutos de transporte de petróleo e derivados com a finalidade de limpar e identificar problemas estruturais nos mesmos (Berto \& Lourenço, 2006) tais como: incrustações ou formação de sedimentos e a corrosão ou desgaste da parede interna (Lima, 2014).

Segundo Diaz (2008), os PIGs são classificados quanto a sua tarefa, os de limpeza (Cleaning PIGs) são utilizados na limpeza e secagem dos dutos, enquanto os de inspeção (Smart PIGs) realizam o trabalho de sensoriamento interno da tubulação e é justamente nesse tipo de PIG que se concentra o foco deste trabalho.

Para a detecção dos problemas citados, o PIG percorre o duto de forma autônoma através do diferencial de pressão (Pereira, Oliveira, Lima, Salazar, Maitelli, \& Fontes, 2013). Esse deslocamento deve atender à especificação para velocidade média de $3 \mathrm{~m} / \mathrm{s}$, conforme Sadovnychiy, López, Ponomaryov, $\&$ Sadovnychyy (2006), para garantir uma boa inspeção. Acontece que, em alguns casos ao deparar-se com algum tipo de obstrução, o PIG emperra no duto. Depois disso, a pressão atrás do PIG (à montante) aumenta de forma drástica e ao vencer tal obstáculo o PIG atinge velocidades elevadas e indesejadas que impossibilitam o correto sensoriamento. Esse fenômeno é denominado pelo jargão técnico de "tiro", fazendo-se necessário um sistema eletromecânico capaz de controlar a velocidade desses instrumentos.

Este trabalho faz parte dessa proposta para o controle das altas velocidades atingidas por PIGs instrumentados durante o fenômeno de "tiro" e traz detalhes do desenvolvimento de um tacômetro magnético para medir a velocidade desses PIGs. A escolha do material de origem magnética se deu pela inerência dele ao meio onde se executam as medições, trazendo mais robustez ao sistema.

É importante ressaltar o estado da arte ao qual se encontram os estudos tomados como base para a escrita deste documento, como Silva \& Pinho (2001) e Sadovnychiy et al. (2006). Nestes trabalhos é comum a menção da palavra odômetro para denominar a parte do PIG encarregada de medir a distância percorrida por eles dentro das tubulações. Neste trabalho, o termo tacômetro é introduzido, diante da necessidade de se mensurar o quão rápido esse movimento ocorre através da medição de sua velocidade, para que o controle possa ser realizado. 


\section{REVISÃO BIBLIOGRÁFICA}

\subsection{Efeito Hall}

Em 1879, o físico norte americano Edwin $\mathrm{H}$. Hall observou a força que age em um material semicondutor transportando uma corrente, quando exposto a um campo magnético transversal. Notou que há uma deflexão dos elétrons, resultante da força magnética (Pinto, 2010), conforme a Figura 1.

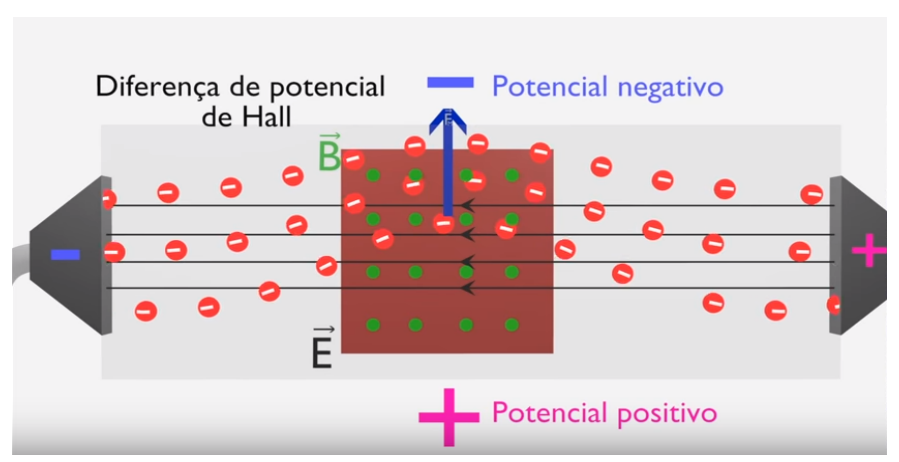

Figura 1: Material semicondutor sob a ação de um campo magnético.

Essa deflexão causa um acúmulo de cargas negativas em uma das extremidades do material e a ausência delas na outra, gerando uma diferença de potencial, diminuindo a velocidade do deslocamento transversal desses elétrons. Esse efeito ficou conhecido como efeito Hall em sua homenagem.

\subsection{Sensor de efeito Hall}

Segundo Braga (2015), o sensor de efeito Hall é um dispositivo capaz de captar esse efeito e responder com uma variação em seu terminal de saída, como mostra a Figura 2.

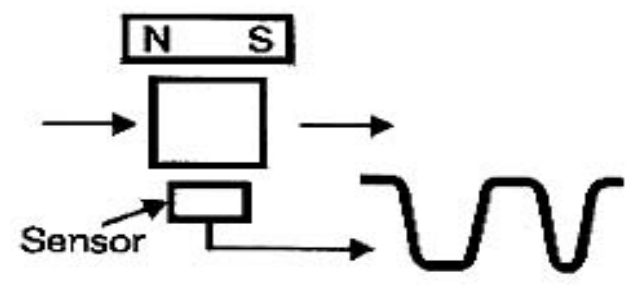

Figura 2: Sensor de efeito Hall sob influência de um campo magnético.

Ainda segundo Braga (2015), podemos detectar o movimento de uma peça prendendo um ímã à sua superfície e dessa forma medir sua rotação, seguindo o esquema da Figura 3. 


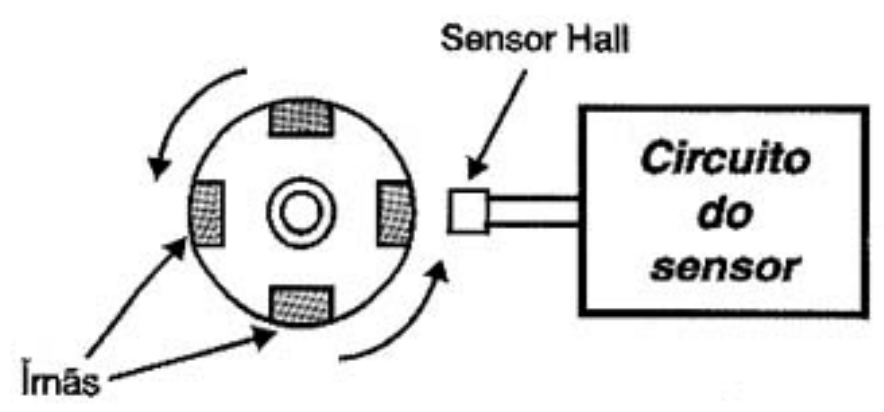

Figura 3: Circuito detector de variação de campo magnético.

\section{3 Ímãs de Neodímio}

O efeito Hall é de extrema importância para os circuitos eletromagnéticos, e funcionam em conjunto com os ímãs para se obter variações de tensão.

Em 1983, foram descobertos os ímãs baseados em neodímio, ou "super ímãs" apelidados dessa maneira por terem o mais elevado campo magnético dentre todos os tipos de ímãs (Botelho, 2008). Apesar de seu custo elevado, podem ser facilmente encontrados no cabeçote de posicionamento do leitor dos drivers de DVD. Com o constante descarte desses equipamentos eletrônicos, esses drivers são de fácil aquisição. Os ímãs de neodímio por terem tamanho reduzido, facilitam sua instalação na roda de $66 \mathrm{~mm}$ do protótipo construído e por essa razão foram escolhidos.

Com seu forte campo magnético, espera-se que a deflexão dos elétrons seja forte ao ponto de interromper o fluxo transversal dos mesmos por um curto período, ocasionando um pulso no sinal de saída do sensor, que posteriormente deve ser captado pelo microcontrolador ATMEGA328P.

\subsection{Cálculo da Velocidade}

Para Halliday, Resnick, \& Walker (2009), a velocidade média (Vméd), é a razão entre o deslocamento $(\Delta \mathrm{x})$ e o intervalo de tempo $(\Delta \mathrm{t})$, conforme a Equação 1.

$$
V_{\text {méd }}=\frac{\Delta x}{\Delta t}=\frac{x_{2}-x_{1}}{t_{2}-t_{1}}
$$

Adaptando a Equação (1) para o movimento circular, situação do tacômetro estudado, essa velocidade é conhecida como velocidade linear e pode ser obtida de maneira análoga quando se trata do deslocamento como o comprimento da circunferência " $C$ ", que equivale a $2 \pi r$, conforme a Equação 2.

$$
V_{m e ́ d}=\frac{C}{\Delta t}=\frac{2 \pi r}{t_{2}-t_{1}}
$$

Outra expressão bastante utilizada nesse trabalho é a de conversão de velocidade em metros por segundo (m/s) para rotações por minuto (RPM), 
sabendo da relação que uma circunferência completa equivale a $2 \pi$ radianos [Halliday et al., 2009], temos:

$1 R P M=2 \pi \mathrm{rad} / \mathrm{min}=2 \pi / 60 \mathrm{rad} / \mathrm{s}$

Como $2 \pi / 60=0,10472$ chegamos à Equação 4, utilizada no código implementado para o microcontrolador.

$V_{\text {méd }}=R P M \cdot$ raio $\cdot 0,10472$

\section{METODOLOGIA}

\subsection{Montagem do Protótipo e da Bancada de Testes}

Adquiriu-se uma roda de $66 \mathrm{~mm}$ de diâmetro com rolamento para servir como disco do tacômetro e nela foi fixou-se o ímã de neodímio. Em seguida, soldou-se um segmento de tubo, usinado com a medida de $60 \mathrm{~mm}$ de comprimento e $42 \mathrm{~mm}$ de diâmetro, para encaixe na bancada de testes, como ilustrado na Figura 4.

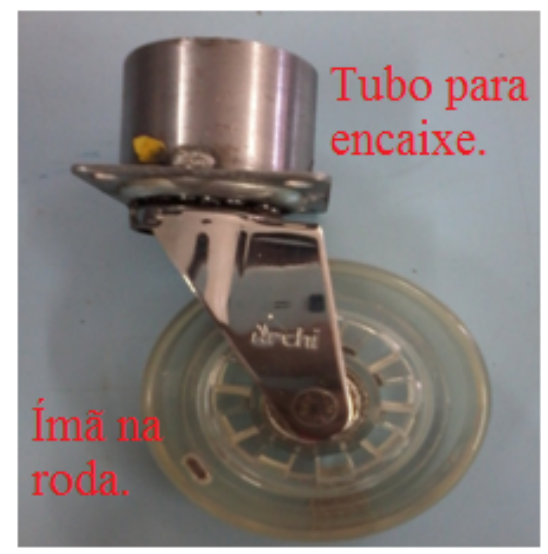

Figura 4: Protótipo do tacômetro com ímã fixado.

Por fim, montou-se uma placa de circuito impresso de tamanho reduzido, para acomodar o sensor e este poder detectar passagem do ímã. Quando esté passar aciona-se a interrupção externa do microcontrolador, que calcula a velocidade. Pode-se visualizar esta placa em detalhe na Figura 5.

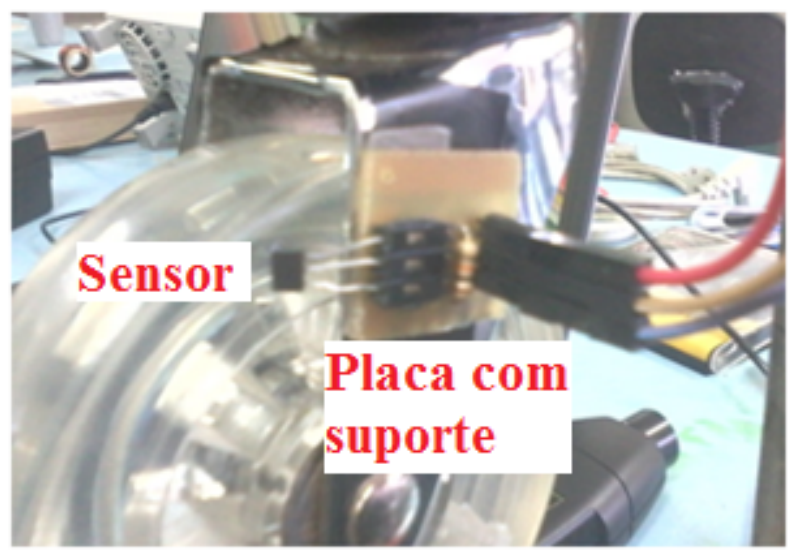

Figura 5: Placa desenvolvida para o sensor Hall. 
Para promover o giro da roda, simulando o movimento de um PIG instrumentado, colocou-se o mandril de um furadeira em contato direto com a roda. Sendo, assim, transmitiu-se a rotação do mandril para a roda. Pode-se visualizar a bancada de testes montada na Figura 6 .

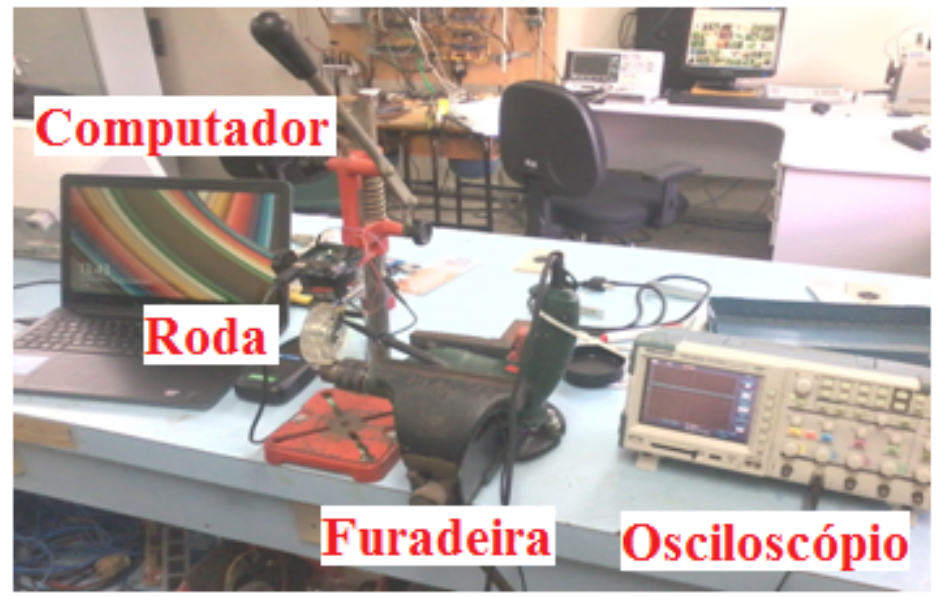

Figura 6: Bancada de testes.

Ainda na Figura 6 , pode-se observar o osciloscópio à direita para o monitoramento do sinal do sensor e o computador à esquerda para a visualização do cálculo da velocidade realizado pelo microcontrolador.

\subsection{Validação}

Com o auxílio de um osciloscópio TPS 2024 da Tektronix, pode-se monitorar o sinal na saída do sensor Hall, conforme a Figura 7, onde cada pulso representa uma passagem do ímã pelo sensor.

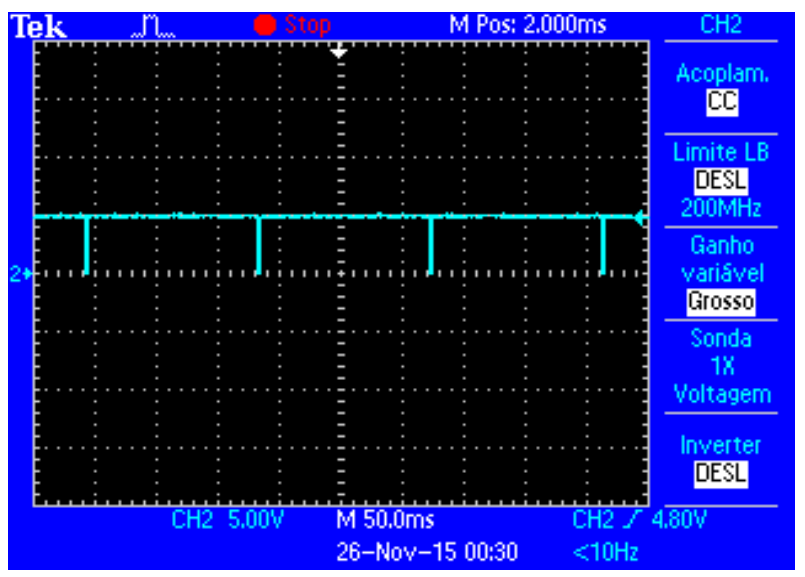

Figura 7: Sinal do sensor Hall, medido a 450 RPM.

\section{RESULTADOS E DISCUSSÕES}

A velocidade máxima, dos testes, alcançada pela roda foi de aproximadamente 1.000 RPM que equivale a $3,5 \mathrm{~m} / \mathrm{s}$. Lembrando que para atender as especificações para uma boa inspeção dos dutos, o PIG instrumentado deve manter uma velocidade média de $3 \mathrm{~m} / \mathrm{s}$. 
Executou-se três testes dentro da faixa de valores possíveis e seus valores de medição estão dispostos na Tabela 1.

Tabela 1: Resultado dos testes com o protótipo.

\begin{tabular}{c|c|c|c}
\hline $\begin{array}{c}\text { oo do } \\
\text { Teste }\end{array}$ & $\begin{array}{c}\text { Tacômet } \\
\text { ro } \\
\text { Óptico } \\
\text { (RPM) }\end{array}$ & $\begin{array}{c}\text { Tacômetr } \\
\text { o } \\
\text { Magnétic } \\
\text { o (RPM) }\end{array}$ & $\begin{array}{c}\text { Erro } \\
\text { Relativo } \\
(\%)\end{array}$ \\
\hline 1 & 419,6 & 420,1 & 0,11916 \\
2 & 511,7 & 512,8 & 0,21496 \\
3 & 967,7 & 969,3 & 0,16534 \\
\hline
\end{tabular}

Com base na Tabela 1 observa-se uma excelente aproximação de valores entre o tacômetro magnético desenvolvido neste trabalho com o tacômetro óptico que serviu como parâmetro de controle. Os valores de erro relativo, apresentados acima, confirmam que foi bem sucedida a montagem do protótipo proposto.

Por fim, filmou-se um dos testes para comprovar a veracidade dos dados expostos na Tabela 1 e pode-se visualizar em:

https://www.youtube.com/watch?v=sPgAY88FGB0 ou https://youtu.be/MM_limml14.

\section{CONCLUSÃO}

Este trabalho teve como enfoque o estudo e desenvolvimento de um tacômetro magnético utilizando um microcontrolador ATMEGA328P e um sensor de efeito Hall modelo 3144, com a finalidade de ser embarcado em um PIG instrumentado para a medição de sua velocidade dentro de dutos de petróleo e gás natural.

Através dos testes em bancada, percebemos que o protótipo respondeu de forma satisfatória e apresentou erros relativos inferiores à faixa de $1 \%$ quando comparados a um tacômetro óptico digital, que serviu de parâmetro de controle.

Esses resultados comprovam o sucesso do desenvolvimento de um tacômetro magnético, que atende as necessidades previamente estabelecidas e consegue medir com precisão a velocidade de um PIG instrumentado.

\section{REFERÊNCIAS}

Berto, C. A. \& Lourenço, S. R. (2006). Sistema de Monitoramento da Corrosão Interna de Gasoduto por Meio de Processamento Digital de Sinais, Revista Exacta, Vol. 4, São Paulo, Brasil, n. 2, p. 391 - 400.

Botelho, I. J. (2008). Modelagem e Controle Linear de um Sistema de Levitação de Ímã Permanente. 137 p. Dissertação de Mestrado, Universidade de São Paulo, Curso de Engenharia de Sistemas. São Paulo-SP. 
Braga, N. C. (2015). Como Funcionam os Sensores de Efeito Hall. Disponível em: http://www.newtoncbraga.com.br/index.php/como-funciona/6640-comofuncionam-os-sensores-de-efeito-hall-art1050. Acesso em: 26/11/2015.

Diaz, J. F. A. (2008). Estudo e Projeto Conceitual de um Robô para Inspeção de Linhas de Serviço. 97 p. Dissertação de Mestrado, Universidade Federal do Rio de Janeiro, Curso de Ciências Mecânicas. Rio de Janeiro, RJ.

Halliday, D., Resnick, R. \& Walker, J. (2009). Fundamentos de Física: Mecânica, LTC - Livros Técnicos e Científicos Editora S.A, 8. ed. Rio de Janeiro, Brasil.

Lima, G. F. Proposta de Tecnologia para Controle de Velocidade de PIGs Instrumentados Utilizando Lógica Fuzzy, 2014, 69 p. Dissertação de Mestrado, Universidade Federal do Rio Grande do Norte, Programa de Pós-Graduação em Engenharia Elétrica e de Computação, Natal-RN.

Pereira, C.G.; Oliveira, R. R. G.; Lima, G. F.; Salazar, A. O.; Maitelli, A. L. \& Fontes, F. A.O. (2013). Proposta de Tecnologia para Controle de Velocidade de PIGs Instrumentados, em 'INTERCON 2013', Trujillo, Perú.

Pinto, J. F. (2010). Magnometria por Efeito Hall, 76 p. Dissertação de Mestrado, Universidade Federal de Pernambuco, Curso de Física, Recife-PE. Sadovnychiy, S.; López, J.; Ponomaryov, V. \& Sadovnychyy, A. (2006). Evaluation of Distance Measurement Accuracy by Odometer for Pipelines PIGs, In Journal of the Japan Petroleum Institute, Vol. 49, no 1, p. $38-42$.

Silva, J. A. P. \& Pinho, A. F. (2001). Ferramenta para Inspeção Geométrica de Dutos de 3", 2001, em Brazilian Petroleum and Gas Institute - IBP, Rio de Janeiro, Brasil, p. 1 - 8. 